\title{
Anti-inflammatory potential of total saponins derived from the roots of Panax ginseng in lipopolysaccharide-activated RAW 264.7 macrophages
}

\author{
KYUNG-JUN JANG ${ }^{1}$, SANG HOON CHOI ${ }^{1}$, GYEONG JIN YU ${ }^{2}$, SU HYUN HONG ${ }^{2}$, YOON HO CHUNG ${ }^{3}$,

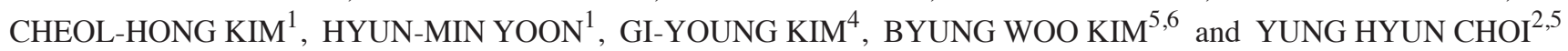

Departments of ${ }^{1}$ Acupuncture and Moxibustion and ${ }^{2}$ Biochemistry, Dongeui University College of Korean Medicine, Busan 614-052; ${ }^{3}$ NamHaeGun Black Garlic Co., Ltd., Busan 614-853; ${ }^{4}$ Laboratory of Immunobiology, Department of Marine Life Sciences, Jeju National University, Jeju 690-756; ${ }^{5}$ Anti-Aging Research Center and Blue-Bio Industry RIC,

College of Natural Sciences and Human Ecology, Dongeui University, Busan 614-714;

${ }^{6}$ Department of Life Science and Biotechnology, Dongeui University, Busan 614-052, Republic of Korea

Received January 22, 2015; Accepted October 29, 2015

DOI: $10.3892 /$ etm.2015.2965

\begin{abstract}
Ginseng, the root of Panax ginseng C.A. Meyer (Araliaceae), is a widely known traditional medicine that has been utilized throughout Asia for several thousand years. Ginseng saponins exert various important pharmacological effects regarding the control of a number of diseases. The aim of the present study was to identify the anti-inflammatory effects of total saponins extracted from ginseng (TSG) on lipopolysaccharide (LPS)-stimulated mouse RAW 264.7 macrophages. The inhibitory effects of TSG on LPS-induced nitric oxide (NO) production and LPS-induced tumor necrosis factor- $\alpha$ (TNF- $\alpha$ ) and interleukin- $1 \beta$ (IL-1 $\beta$ ) protein expression were determined by measuring the levels of nitrite and enzyme-linked immunosorbent assays, respectively. Furthermore, the effects of TSG on the mRNA expression levels and localizations of inducible NO synthase (iNOS), IL- $1 \beta$ and TNF- $\alpha$, and their upstream signaling proteins, including nuclear factor- $\kappa \mathrm{B}(\mathrm{NF}-\kappa \mathrm{B})$ and mitogen-activated protein kinases (MAPKs), were investigated by reverse transcription-polymerase chain reaction and western blotting, respectively. Following stimulation with LPS, elevated levels of NO production were detected in RAW 264.7 cells; however, TSG pretreatment significantly inhibited the production of NO $(\mathrm{P}<0.05)$, by suppressing the expression of iNOS. In addition, LPS-stimulated TNF- $\alpha$ and IL- $1 \beta$ production was significantly reduced by TSG $(\mathrm{P}<0.05)$. In the LPS-stimulated RAW 264.7 cells, NF- $\kappa \mathrm{B}$ was translocated from the cytosol to the nucleus,
\end{abstract}

Correspondence to: Dr Yung Hyun Choi, Department of Biochemistry, Dongeui University College of Korean Medicine, 52-57 Yangjeong-ro, Busanjin-gu, Busan 614-052, Republic of Korea E-mail: choiyh@deu.ac.kr

Key words: ginseng, total saponins, anti-inflammation, nuclear factor- $\kappa \mathrm{B}$, mitogen-activated protein kinases whilst TSG pretreatment induced the sequestration of $\mathrm{NF}-\kappa \mathrm{B}$ in the cytosol by inhibiting inhibitor of $\kappa \mathrm{B}$ degradation. TSG also contributed to downregulation of MAPKs in LPS-stimulated RAW 264.7 cells. These results suggested that TSG may exert anti-inflammatory activity, and that TSG may be considered a potential therapeutic for the treatment of inflammatory diseases associated with macrophage activation.

\section{Introduction}

Inflammation is of importance in the highly complex immune response mounted to defend against harmful stimuli, including pathogens, damaged cells or irritants. Macrophages, as critical participants in the inflammatory process, directly counteract the aforementioned stimuli $(1,2)$. Under inflammatory conditions, enzymes, cytokines and chemokines, in addition to signaling proteins at the site of the infected tissues and cells, are secreted by macrophages, causing inflammatory cells to migrate to sites of inflammation in order to resolve the abnormal conditions $(1,2)$. The model most commonly used to investigate induced inflammation is the stimulation of macrophages by lipopolysaccharide (LPS) obtained from gram-negative bacteria (1). The binding of LPS to its cognate receptors, including Toll-like receptors on the mammalian cell surface, activates several signaling cascades that drive the expression of pro-inflammatory mediators and cytokines, including nitric oxide (NO), prostaglandin $\mathrm{E}_{2}$, tumor necrosis factor- $\alpha$ (TNF- $\alpha$ ) and interleukin (IL)- $1 \beta(2,3)$.

Additionally, excessive inflammatory responses may result in decreased expression of anti-inflammatory cytokines $(1,2,4,5)$. The pathogenesis of autoimmune diseases, which are characterized by chronic inflammation, may be influenced by pro- and anti-inflammatory factors, as well as dysregulation of inflammatory immune responses $(5,6)$. Therefore, the utilization of different therapeutic strategies may be therapeutically beneficial for the treatment of inflammatory diseases; for example, bioactive agents could be used to inhibit the production of inflammatory factors by macrophages (4-6). 
Subsequent to stimulation with LPS, nuclear factor- $\kappa \mathrm{B}$ $(\mathrm{NF}-\kappa \mathrm{B})$ is activated as a result of the activation of the inhibitor of $\kappa \mathrm{B}(\mathrm{I} \kappa \mathrm{B})$-kinase complex inhibitor $(7,8)$. Inhibitor of $\mathrm{I} \kappa \mathrm{B}$ phosphorylates $\mathrm{I} \kappa \mathrm{B}$, causing $\mathrm{I} \kappa \mathrm{B}$ proteasomal degradation and the release of $\mathrm{NF}-\kappa \mathrm{B}(7,8)$. The liberated dimeric $\mathrm{NF}-\kappa \mathrm{B}$ then translocates to the nucleus and activates the transcription of pro-inflammatory target genes that encode regulatory proteins. This leads to physiological responses, including inflammatory or immune responses $(7,8)$. Downstream targets of the LPS-induced inflammatory cascades in macrophages are members of the mitogen-activated protein kinase (MAPK) family, including extracellular signal-regulated kinase (ERK), c-Jun $\mathrm{NH}_{2}$-terminal kinase (JNK) and p38 MAPK $(2,3,7)$. When macrophages are stimulated with LPS, MAPKs are activated to produce inflammatory factors through the activation of multiple downstream signaling events $(9,10)$. Therefore, targeting the NF- $\mathrm{NB}$ and MAPK signaling pathways is considered an attractive therapeutic strategy for the development of anti-inflammatory therapeutic agents.

Ginseng, the root of Panax ginseng C.A. Meyer (Araliaceae), is a herbal medicine that has been shown to exhibit a variety of therapeutic effects, including neuroprotective, immunomodulating, anti-cancer and antioxidant activities (9). As part of traditional folk medicine, ginseng has been a popular plant-based medicine for 2,000 years in East Asian countries, and remains a popular natural medicine worldwide (11-13). The major active constituents of ginseng are the triterpenoid saponins, also known as total saponins extracted from ginseng (TSG), which have a four-ring, steroid-like structure with attached sugar moieties (14-16). TSG are the primary molecules responsible for the effects of ginseng. They possess pharmacological properties, including anti-inflammatory, antioxidant and anti-cancer effects (11-13). In addition, Kim et al (17) suggested that TSG may suppress NO production in LPS/interferon- $\gamma$-activated RAW 246.7 macrophages by inhibiting inducible NO synthase (iNOS) expression. Furthermore, TSG has been found to inhibit LPS-induced expression of iNOS, matrix metalloproteinase-9 and pro-inflammatory cytokines in BV2 microglial cells, which is associated with the inactivation of NF- $\mathrm{B}$ and MAPK signaling pathways (18). A recent study also demonstrated that TSG-associated recovery from LPS-induced depression-like behavior was associated with decreased production of various pro-inflammatory cytokines in LPS-challenged mice and RAW 264.7 cells (19). However, the anti-inflammatory mechanisms in macrophages have yet to be elucidated.

Therefore, in the present study, the anti-inflammatory effects of TSG were evaluated using an LPS-stimulated RAW 264.7 macrophage cell model. It was identified that TSG exerted curative effects on anti-inflammatory activity by reducing iNOS production, and TNF- $\alpha$ and IL- $1 \beta$ expression. The effects of TSG on the NF- $\kappa \mathrm{B}$ and MAPK signaling pathways were also investigated in the current study in order to elucidate the associated inhibitory mechanism. The results provide evidence in favor of the use of TSG as a potential anti-inflammatory supplement.

\section{Materials and methods}

Preparation of TSG. For the preparation of TSG, air-dried ginseng roots were purchased from Dongeui University
Hospital of Oriental Medicine (Busan, Korea). TSG was separated and purified as described by Sugimoto et al (20). Briefly, samples were extracted twice with methanol (Sigma-Aldrich, St. Louis, MO, USA) by refluxing at $80^{\circ} \mathrm{C}$ for $2 \mathrm{~h}$. The methanol extract was then suspended in water and partitioned sequentially with n-hexane, chloroform, ethyl acetate and n-butanol. Subsequently, the water-saturated n-butanol fraction was evaporated to dryness in a vacuum. The recovered crude saponins were loaded onto Diaion ${ }^{\circledR}$ HP-20/MCI GEL ${ }^{\circledR}$ CHP20P (Sigma-Aldrich), and the sugar residues were removed with $40 \%$ methanol. The fractions were eluted with $60-80 \%$ methanol, collected, and dried to obtain TSG. TSG was then diluted in Dulbecco's modified Eagle medium (DMEM; Gibco; Thermo Fisher Scientific, Inc., Waltham) MA, USA) to $0,50,100,200$ and $400 \mu \mathrm{g} / \mathrm{ml}$, prior to use.

Cell culture. Mouse RAW 264.7 macrophages were purchased from the American Tissue Culture Collection (Manassas, VA, USA). RAW 264.7 cells $\left(5 \times 10^{5}\right.$ cells $\left./ \mathrm{ml}\right)$ were grown in DMEM supplemented with $10 \%$ fetal bovine serum and $1 \%$ (v/v) penicillin $(100 \mathrm{U} / \mathrm{ml}) /$ streptomycin $(100 \mu \mathrm{g} / \mathrm{ml})$ under humidified conditions $\left(5 \% \mathrm{CO}_{2}\right.$ at $\left.37^{\circ} \mathrm{C}\right)$. In all experiments, the cells were treated with various concentrations of TSG $(0,50,100,200$ or $400 \mu \mathrm{g} / \mathrm{ml})$ for $1 \mathrm{~h}$ prior to exposure with $100 \mathrm{ng} / \mathrm{ml}$ LPS (Lipopolysaccharides from Escherichia coli 026:B6; Sigma-Aldrich) for 24 h under humidified conditions $\left(5 \% \mathrm{CO}_{2}\right.$ at $\left.37^{\circ} \mathrm{C}\right)$.

3-(4,5-dimethylthiazol-2-yl)-2,5-diphenyl-tetrazolium bromide (MTT) assay. Cell viability was measured based on the formation of blue formazan, which is metabolized from colorless MTT (Sigma-Aldrich) by mitochondrial dehydrogenases, enzymes that are only active in live cells (18). RAW 264.7 cells $\left(5 \times 10^{5}\right.$ cells $\left./ \mathrm{ml}\right)$ were seeded in a 96-well plate. The cells were pretreated with various concentrations of TSG for $1 \mathrm{~h}$ and then stimulated by $100 \mathrm{ng} / \mathrm{ml}$ LPS for $24 \mathrm{~h}$. Following incubation with TSG and LPS, the cultured media was replaced with fresh media and the cells were incubated with $0.5 \mathrm{mg} / \mathrm{ml} \mathrm{MTT}$ solution for $3 \mathrm{~h}$. The supernatant was then discarded and the formazan blue, which was formed in the cells, was dissolved with dimethyl sulfoxide (Sigma-Aldrich). The optical density was measured at $540 \mathrm{~nm}$ with an ELISA plate reader (MRX; Dynatech Laboratories, Chantilly, VA, USA). Cell viability was calculated as the percentage of surviving cells over control cells (no TSG added).

Measurement of NO production. Concentrations of NO in the culture supernatants were determined by measuring the levels of nitrite, which is a major stable product of NO, using Griess reagent (Sigma-Aldrich). RAW 264.7 cells $\left(5 \times 10^{5}\right.$ cells $\left./ \mathrm{ml}\right)$ were seeded in each well of a 96-well plate. The cells were pretreated with the indicated concentrations of TSG for $1 \mathrm{~h}$ and stimulated with $100 \mathrm{ng} / \mathrm{ml}$ LPS. Following an incubation period of $24 \mathrm{~h}$, the supernatant from each well was collected by centrifugation at 3,000 x g using the Smart R17 Refrigerated Micro Centrifuge (Hanil BioMed, Inc., Gwangju, Korea) for $20 \mathrm{~min}$ at $4^{\circ} \mathrm{C}$. The supernatant was mixed with the same volume of Griess reagent for $10 \mathrm{~min}$ at room temperature in the dark. Nitrite levels were determined using an ELISA plate reader (MRX; Dynatech Laboratories) at $540 \mathrm{~nm}$, and nitrite concentrations 
were calculated by referencing a standard curve generated by known concentrations of sodium nitrite (17).

Measurement of TNF- $\alpha$ and IL-1 $\beta$ production. To measure the inhibitory efficacy of TSG on the production of TNF- $\alpha$ and IL- $1 \beta$, TNF- $\alpha$ and IL-1 $\beta$ ELISA kits (cat nos. MTA00B and MLB00C, respectively) were purchased from R\&D Systems, Inc. (Minneapolis, MN, USA). The cell culture conditions were the same as for the nitrite measurement assay. After incubation with TSG and LPS for $24 \mathrm{~h}$, the TNF- $\alpha$ and IL- $1 \beta$ concentrations in cultured media were determined by a selective ELISA kit, according to the manufacturer's protocol.

Reverse transcription-polymerase chain reaction (RT-PCR). The cells were incubated with TSG (400 $\mu \mathrm{g} / \mathrm{ml})$ alone for $24 \mathrm{~h}$, or pretreated with the various concentrations of TSG for $1 \mathrm{~h}$ prior to LPS stimulation $(100 \mathrm{ng} / \mathrm{ml})$ for $24 \mathrm{~h}$. Total RNA was isolated from cultured cells using TRIzol ${ }^{\circledR}$ reagent (Invitrogen; Thermo Fisher Scientific). Contaminating DNA was removed by treating the RNA samples with recombinant DNase I (DNA-free ${ }^{\mathrm{TM}}$ kit; Thermo Fisher Scientific, Inc.) The isolated RNA $(1 \mu \mathrm{g})$ was used for cDNA synthesis using AccuPower ${ }^{\circledR}$ RT PreMix (Bioneer Corporation, Daejeon, Korea) containing Moloney murine leukemia virus reverse transcriptase. iNOS, IL-1 $\beta$ and TNF- $\alpha$ genes were amplified from the cDNA by PCR (5331 MasterCycler Gradient; Eppendorf, Hamburg, Germany). The PCR primers were as follows: iNOS forward, 5'-ATGTCCGAAGCAAACATCAC-3' and reverse, 5'-TAA TGTCCAGGAAGTAGGTG-3'; IL-1 $\beta$ forward, 5'-GGGCTG CTTCCAAACCTTTG-3' and reverse, 5'-GCTTGGGAT CCACACTCTCC-3'; TNF- $\alpha$ forward, 5'-TCTCATCAGTTC TATGGCCC-3' and reverse, 5'-GGGAGTAGACAAGGT ACAAC-3'; and glyceraldehyde 3-phosphate dehydrogenase (GAPDH) forward, 5'-AGGCCGGTGCTGAGTATGTC-3' and reverse, 5'-TGCCTGCTTCACCACCTTCT-3' (Bioneer Corporation). The PCR reaction was initiated at $94^{\circ} \mathrm{C}$ for $2 \mathrm{~min}$, followed by 31 cycles of $94^{\circ} \mathrm{C}$ for $30 \mathrm{sec}, 30 \mathrm{sec}$ annealing temperature, $72^{\circ} \mathrm{C}$ for $30 \mathrm{sec}$, and a final extension step at $72^{\circ} \mathrm{C}$ for $5 \mathrm{~min}$. The annealing temperatures were $63^{\circ} \mathrm{C}$ for iNOS, IL- $1 \beta$ and TNF- $\alpha$, and $61^{\circ} \mathrm{C}$ for GAPDH. Following amplification, the PCR products were separated by $1.5 \%$ agarose gel electrophoresis, stained with ethidium bromide (Sigma-Aldrich) and visualized by ultraviolet illumination.

Protein extraction and western blot analysis. For total protein extraction, the cells were lysed by incubating with a lysis buffer [25 mM Tris- $\mathrm{Cl}$ ( $\mathrm{pH} 7.5), 250 \mathrm{mM} \mathrm{NaCl}, 5 \mathrm{mM}$ ethylene diaminetetra acetic acid, 1\% NP-40, 1 mM pheny-methylsulfonyl fluoride and $5 \mathrm{mM}$ dithiothreitol; Sigma-Aldrich] for $1 \mathrm{~h}$ at $4^{\circ} \mathrm{C}$. Insoluble materials were discarded by centrifugation at 13,000 x g using the Smart R17 Refrigerated Micro Centrifuge (Hanil BioMed, Inc.) for $20 \mathrm{~min}$ at $4^{\circ} \mathrm{C}$. In a parallel experiment, nuclear and cytosolic proteins were separated using nuclear extraction reagents (product no. 78833; Pierce Biotechnology, Inc., Rockford, IL, USA), according to the manufacturer's protocol. The protein concentration in the cell lysate was determined using a DC ${ }^{\text {Tм }}$ Protein Assay (Bio-Rad Laboratories, Inc., Hercules, CA, USA). Equal quantities of protein were then separated by $10 \%$ sodium dodecyl sulfate-polyacrylamide gel electrophoresis at $90 \mathrm{~V}$ for $2 \mathrm{~h}$. Separated protein was transferred to a nitrocellulose membrane (Schleicher and Schuell, Keene, NH, USA) and subsequently blocked with Tris-buffered saline (10 mM of Tris- $\mathrm{Cl}, \mathrm{pH} 7.4$ ) containing $0.5 \%$ Tween-20 (TBST) and 5\% nonfat dried milk for $1 \mathrm{~h}$ at room temperature. Subsequently, the membranes were probed with rabbit anti-iNOS (1:1,000; sc-509), -IL-1 $(1: 1,000 ;$ sc-7884), -p65 (1:500; sc-109), -IкB- $\alpha$ (1:500; sc-847), -ERK (1:1,000; sc-154) and -p38 (1:1,000; sc-535), and goat anti-lamin B (1:1,000; sc-6216) and -actin (1:1,000; sc-1615) polyclonal antibodies, from Santa Cruz Biotechnology, Inc. (Dallas, TX, USA), and rabbit anti-TNF- $\alpha$ (1:1,000; \#3707), -JNK (1:1,000; \#9252S) and phosphorylated (p)-38 (1:500; \#9211S) polyclonal antibodies, and mouse anti-p-JNK (1:500; \#9255) and -p-ERK (1:500; \#9106S) monoclonal antibodies, from Cell Signaling Technology, Inc. (Danvers, MA, USA), overnight at $4^{\circ} \mathrm{C}$. After $2 \mathrm{~h}$ blocking with $5 \%$ non-fat milk in TBST $(1.5 \mathrm{M} \mathrm{NaCl}, 20 \mathrm{mM}$ Tris-Cl, $0.05 \%$ Tween-20, pH 7.4), the membranes were incubated with horseradish peroxidase-conjugated anti-rabbit IgG secondary antibodies (1:1,000; RPN4301; GE Healthcare Life Sciences, Chalfont, UK) at room temperature for $2 \mathrm{~h}$. Using an enhanced chemiluminescence detection system (GE Healthcare Life Sciences), immunoreactive bands were detected and exposed to X-ray film.

Statistical analysis. Data are presented as the mean \pm standard deviation. Differences in mean values between groups were analyzed by a one-way analysis of variance followed by Dunnett's-test. $\mathrm{P}<0.05$ was considered to indicate a statistically significant difference.

\section{Results}

TSG inhibits LPS-induced NO production in RAW 264.7 macrophages. To evaluate the anti-inflammatory activity of TSG, the effect of TSG on NO production stimulated by LPS was initially investigated in RAW 264.7 cells. Following pretreatment with various concentrations of TSG for $1 \mathrm{~h}$, RAW 264.7 cells were stimulated with LPS for $24 \mathrm{~h}$. Cell culture media were collected and NO levels were quantified using the Griess test. The addition of LPS to RAW 264.7 cells resulted in an increase in NO production levels (Fig. 1). However, TSG was observed to significantly suppress LPS-induced NO production in a concentration-dependent manner (Fig. 1; $\mathrm{P}<0.05$ ).

TSG downregulates LPS-induced iNOS expression in RAW 264.7 macrophages. Following the observation that NO production was inhibited by TSG, RT-PCR and western blot analysis were performed to determine whether the inhibition of NO production by TSG in the LPS-stimulated RAW 264.7 cells was associated with decreased levels of iNOS mRNA and protein, as iNOS produces NO as a key mediator of inflammation. In response to LPS treatment, mRNA expression of iNOS was induced; however, pretreatment with TSG inhibited this upregulation at a dose of $400 \mu \mathrm{g} / \mathrm{ml}$ (Fig. 2A). Similarly, pretreatment with TSG markedly suppressed the protein expression levels of iNOS in a concentration-dependent manner (Fig. 2B). The results indicate that TSG can downregulate NO production in LPS-stimulated RAW 264.7 cells via inhibition of iNOS expression at the transcriptional level. 


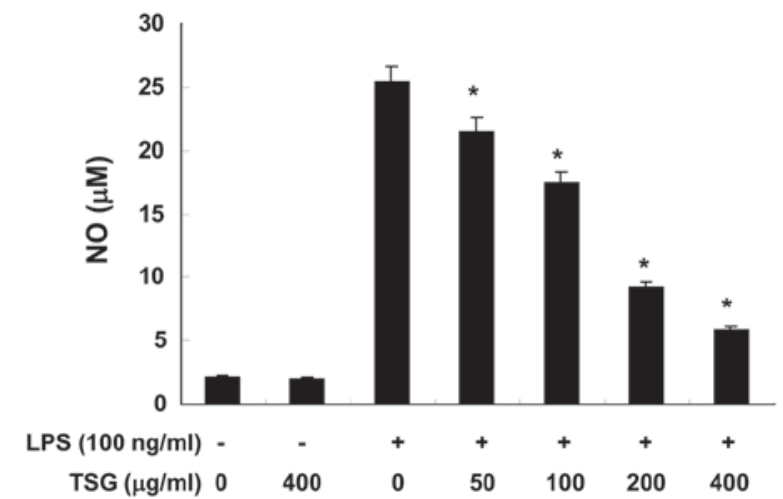

Figure 1. Inhibition of NO production by TSG in LPS-stimulated RAW 264.7 macrophages. RAW 264.7 cells were pretreated with the indicated concentrations of TSG for $1 \mathrm{~h}$ and then stimulated by LPS $(100 \mathrm{ng} / \mathrm{ml})$ for $24 \mathrm{~h}$. NO was measured using Griess reagent. Values are presented as mean \pm standard deviation. " $\mathrm{P}<0.05$ vs. LPS only treatment. NO, nitric oxide; LPS, lipopolysaccharide, TSG, total saponins extracted from ginseng.

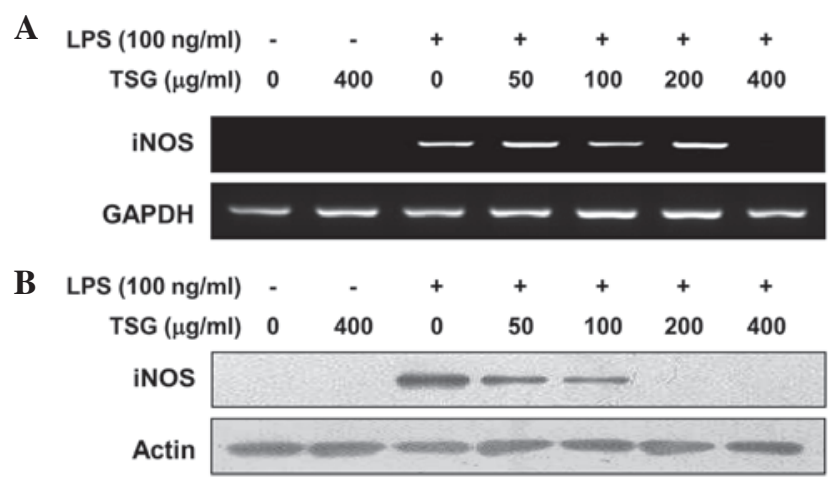

Figure 2. Inhibition of iNOS expression by TSG in LPS-stimulated RAW 264.7 macrophages. RAW 264.7 cells were pretreated with the indicated concentrations of TSG for $1 \mathrm{~h}$ and then stimulated by LPS $(100 \mathrm{ng} / \mathrm{ml})$ for $24 \mathrm{~h}$. (A) mRNA and (B) protein expression levels of iNOS were determined by reverse transcription-polymerase chain reaction and western blot analysis, and GAPDH and actin were used as internal controls, respectively. LPS, lipopolysaccharide; TSG, total saponins extracted from ginseng; iNOS, inducible nitric oxide synthase; GAPDH, glyceraldehyde 3-phosphate dehydrogenase.

Effects of TSG on the viability of RAW 264.7 macrophages. In order to exclude the possibility that the inhibition of NO production was due to cytotoxicity caused by TSG treatment, MTT assays were performed in RAW 264.7 cells treated with TSG for $24 \mathrm{~h}$ in the presence or absence of LPS $(100 \mathrm{ng} / \mathrm{ml})$. As demonstrated in Fig. 3, at the same concentrations of TSG $(50-400 \mu \mathrm{g} / \mathrm{ml})$ used to inhibit NO and its production, TSG alone did not affect cell viability. Furthermore, co-treatment with TSG and LPS also did not demonstrate any cytotoxic effects. Thus, the results indicate that the inhibition of NO production in LPS-stimulated RAW 264.7 cells was not a result of the cytotoxic effects of TSG.

TSG reduces the production of pro-inflammatory cytokines in LPS-stimulated RAW 264.7 macrophages. The effects of TSG on pro-inflammatory cytokines, such as TNF- $\alpha$ and IL-1 $\beta$, were analyzed using ELISA. As demonstrated in Fig. 4A and B, when RAW 264.7 cells were treated with TSG alone, there were no significant changes in the production levels of

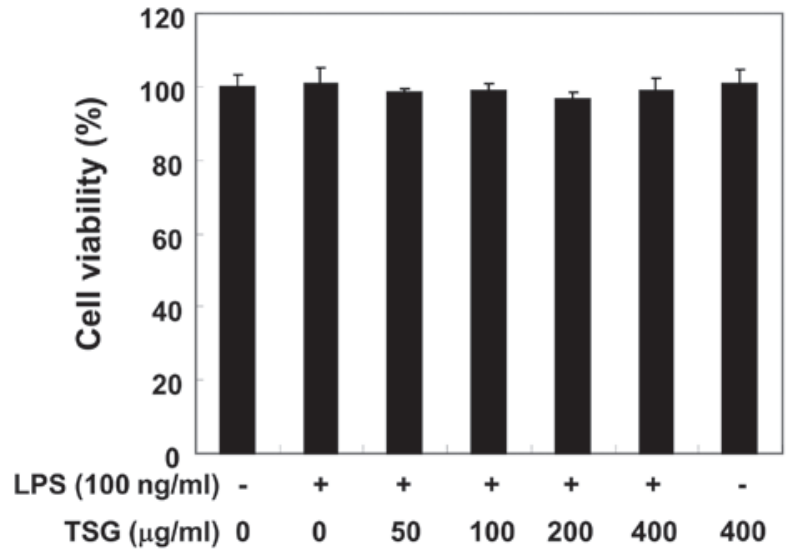

Figure 3. Effects of TSG and LPS on the viability of RAW 264.7 macrophages. Cells were treated with the indicated concentrations of TSG or LPS alone, or treated with TSG for $1 \mathrm{~h}$ prior to treatment with LPS. Following a 24-h incubation period, cell viability was assessed using a 3-(4,5-dimethylthiazol-2-yl)-2,5-diphenyl-tetrazolium bromide reduction assay. Cell viability was calculated as the percentage of surviving cells over control cells (no TSG added). Values are presented as mean \pm standard deviation of three independent experiments. LPS, lipopolysaccharide; TSG, total saponins extracted from ginseng.

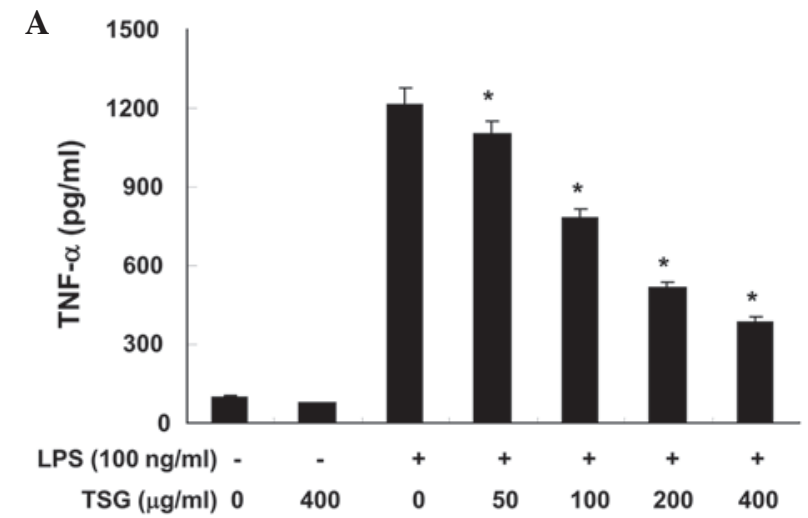

B

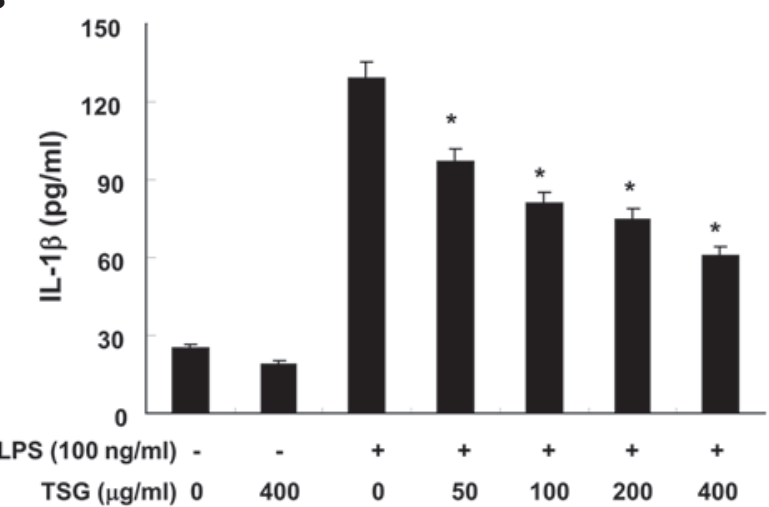

Figure 4. Inhibition of TNF- $\alpha$ and IL-1 $\beta$ production by TSG in LPS-stimulated RAW 264.7 macrophages. RAW 264.7 cells were pretreated with various concentrations of TSG for $1 \mathrm{~h}$ and then stimulated by LPS $(100 \mathrm{ng} / \mathrm{ml})$ for $24 \mathrm{~h}$. (A) TNF- $\alpha$ and (B) IL-1 $\beta$ production were measured using the corresponding ELISA kits. Values are presented as mean \pm standard deviation. ${ }^{*}<<0.05$ vs. LPS only treatment. TNF- $\alpha$, tumor necrosis factor- $\alpha$; LPS, lipopolysaccharide; TSG, total saponins extracted from ginseng; IL- $1 \beta$, interleukin- $1 \beta$.

TNF- $\alpha$ or IL-1 $\beta$, respectively ( $\mathrm{P}>0.05$ ). However, production of the two cytokines were increased in the culture media of LPS-stimulated RAW 264.7 macrophages, and these increases 
A

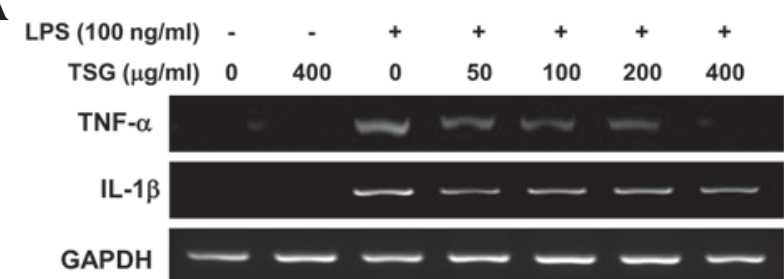

B

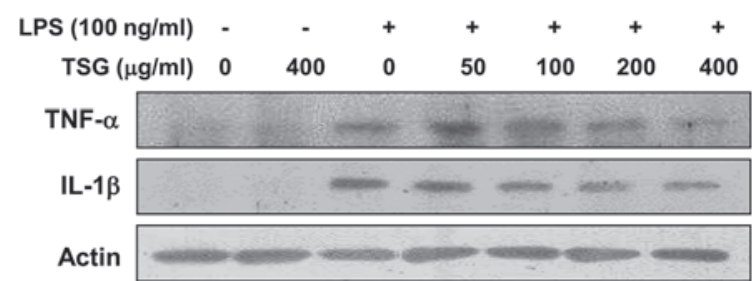

Figure 5. Inhibition of LPS-induced TNF- $\alpha$ and IL-1 $\beta$ expression by TSG in LPS-stimulated RAW 264.7 macrophages. RAW 264.7 cells were pretreated with various concentrations of TSG for $1 \mathrm{~h}$ and then stimulated by LPS $(100 \mathrm{ng} / \mathrm{ml})$ for $24 \mathrm{~h}$. (A) mRNA and (B) protein expression levels of TNF- $\alpha$ and IL-1 $\beta$ were determined by performing reverse transcription-polymerase chain reaction (RT-PCR) and western blot analysis, in which GAPDH and actin were used as internal controls, respectively. LPS, lipopolysaccharide; TSG, total saponins extracted from ginseng; TNF- $\alpha$, tumor necrosis factor- $\alpha$; IL-1 $\beta$, interleukin-1 $\beta$; GAPDH, glyceraldehyde 3-phosphate dehydrogenase.

were significantly reduced by treatment with TSG in a concentration-dependent manner ( $\mathrm{P}<0.05$; Fig. 4).

TSG downregulates LPS-induced TNF- $\alpha$ and IL-1 $\beta$ mRNA and protein expression in RAW 264.7 macrophages. In a parallel experiment, RT-PCR and western blot analyses were performed to determine whether TSG was able to inhibit the expression of TNF- $\alpha$ and IL- $1 \beta$ cytokines at the transcriptional or translational levels. As indicated in Fig. 5, treating RAW 264.7 macrophages with different concentrations of TSG prior to LPS treatment resulted in a dose-dependent decrease in the mRNA (Fig. 5A) and protein (Fig. 5B) levels of the two cytokines. Thus, the results indicate that TSG also inhibits the expression of these genes at the transcriptional level.

TSG blocks LPS-induced NF- $\kappa B$ activity in LPS-stimulated $R A W 264.7$ macrophages. NF- $\mathrm{KB}$ is an important transcription factor that is required for the activation of pro-inflammatory mediators and cytokines in LPS-stimulated macrophages. Under normal conditions, NF- $\mathrm{kB}$ is localized to the cytosol due to its binding with IкB. However, when cells are stimulated by LPS, IкB protein is rapidly phosphorylated by ІкB kinase and degraded, and the NF- $\mathrm{KB}$ is translocated into the nucleus (7). Therefore, to determine whether TSG prevents the translocation of NF- $\mathrm{kB}$ from the cytosol to the nucleus, western blot analysis was performed using the nuclear and cytosolic fractions of RAW 264.7 cells. Fig. 6A demonstrates that the protein expression level of the nuclear p65 subunit of NF- $\kappa B$ was markedly increased following exposure to LPS alone, indicating that LPS induced the translocation of NF- $\mathrm{KB}$ p65 from the cytosol to the nucleus. However, the LPS-induced accumulation of NF- $\kappa \mathrm{B}$ p65 in the nuclear fractions was reduced by TSG pretreatment in a concentration-dependent manner (Fig. 6A). In addition, I $\kappa \mathrm{B}-\alpha$ exhibited minor
A

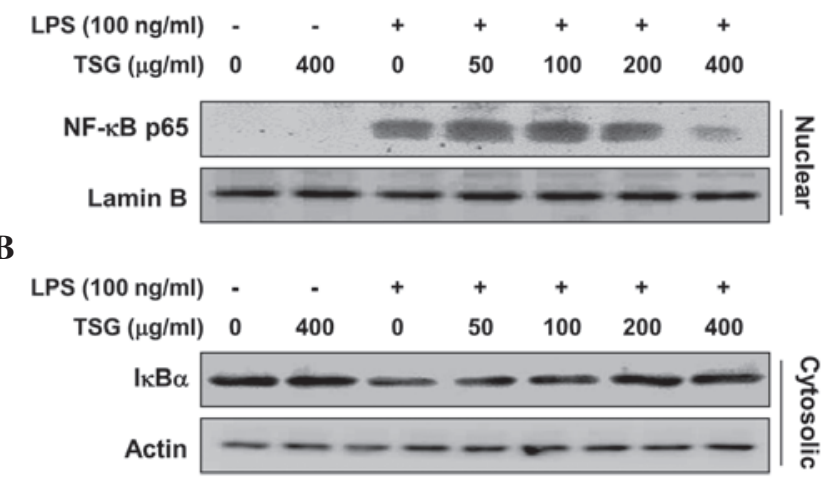

Figure 6. Effects of TSG on LPS-induced NF- $\kappa$ B translocation and expression in RAW 264.7 macrophages. RAW 264.7 cells were pretreated with the indicated concentrations of TSG for $1 \mathrm{~h}$ prior to $100 \mathrm{ng} / \mathrm{ml}$ LPS stimulation

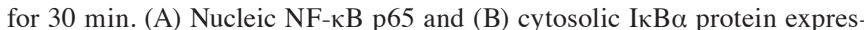
sion were measured using western blotting. Lamin B and actin were used as internal controls for the nuclear and cytosolic fractions, respectively. LPS, lipopolysaccharide; TSG, total saponins extracted from ginseng; $N F-\kappa B$, nuclear factor- $\kappa \mathrm{B}$; I $\kappa \mathrm{B}-\alpha$, inhibitor of $\kappa \mathrm{B}-\alpha$.

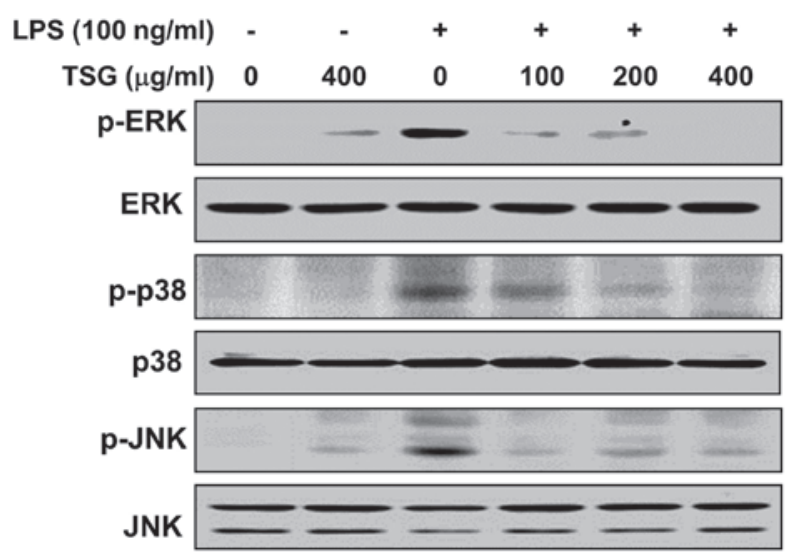

Figure 7. Effects of TSG on the activation of mitogen-activated protein kinases induced by LPS in RAW 264.7 macrophages. Cells were treated with TSG $1 \mathrm{~h}$ prior to treatment with LPS $(100 \mathrm{ng} / \mathrm{ml})$ for $1 \mathrm{~h}$. Total proteins were subjected to $10 \%$ sodium dodecyl sulfate-polyacrylamide gels, followed by western blot analysis using the indicated antibodies. LPS, lipopolysaccharide; TSG, total saponins extracted from ginseng; $\mathrm{p}-$, phosphate-; ERK, extracellular signal-regulated kinase; JNK, c-Jun $\mathrm{NH}_{2}$-terminal kinase; $N F-\kappa B$, nuclear factor- $\kappa \mathrm{B}$; I $\mathrm{B}-\alpha$, inhibitor of $\kappa \mathrm{B}-\alpha$.

degradation following exposure to LPS; however, TSG blocked the LPS-induced degradation of IкB- $\alpha$ in a dose-dependent manner (Fig. 6B). These findings indicate that TSG inhibits NF- $\mathrm{KB}$ activation in RAW 264.7 cells through the suppression

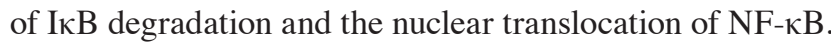

TSG reduces LPS-induced activation of MAPKS in LPS-stimulated RAW 264.7 macrophages. As the MAPK signaling pathway is known to be important for the expression of pro-inflammatory genes, MAPKs act as specific targets for inflammatory responses $(9,10)$. To examine whether inhibition of the LPS-induced inflammatory action by TSG is mediated through MAPK signaling pathways, the effects of TSG on the activation of MAPKs, including p38 MAPK, ERK and JNK, were investigated in LPS-stimulated RAW 264.7 cells. As indicated in Fig. 7, phosphorylation of p38 MAPK, ERK and 
JNK were induced by stimulation with LPS, whereas TSG attenuated the LPS-induced phosphorylation of these kinases in a dose-dependent manner. However, the total protein expression levels of the unphosphorylated MAPKs were unaffected by LPS and TSG treatment. These results suggest that TSG may block the LPS-induced expression of pro-inflammatory responses by inhibiting the MAPK signaling pathway.

\section{Discussion}

The inflammatory process is typically highly regulated, consisting of signals that initiate and maintain inflammation, and signals that inhibit the process $(4,5)$. Due to the importance of activated macrophages in the initiation and amplification of a variety of inflammatory diseases, therapeutic methods to reduce the number of activated macrophages, inhibit their activation signals and/or specific macrophage receptors, or to selectively counteract macrophage products, are under investigation for controlling inflammatory diseases (1). In a previous study, it was demonstrated that TSG was able to exert anti-inflammatory effects in LPS-activated RAW 264.7 macrophages (19). While this is in agreement with the results of the present study, the molecular mechanisms have not been well characterized. The current study also demonstrated that the anti-inflammatory properties of TSG may be associated with a reduction in the activation levels of NF- $\mathrm{KB}$ and MAPK signaling pathways.

$\mathrm{NO}$ is a highly reactive oxidant that is produced through the action of iNOS, and participates in diverse biological mechanisms as a potent pro-inflammatory mediator $(21,22)$. iNOS is present at low levels under normal physiological conditions, however, it is rapidly induced by pro-inflammatory and mitogenic stimuli, including LPS $(2,18)$. Numerous studies have revealed that excessive NO production is important in the pathogenesis of inflammation and can lead to tissue damage by reacting with reactive oxygen species $(23,24)$. Several inhibitors of NO induction have been reported to exert anti-inflammatory effects by preventing iNOS expression $(17,18,22)$. In the present study, TSG was found to significantly reduce the production of NO in LPS-stimulated RAW 264.7 macrophages by inhibiting mRNA and protein levels of iNOS, suggesting that the specific inhibition of the iNOS gene may be responsible for the anti-inflammatory capacity of TSG. An MTT assay confirmed that the inhibitory effects of TSG were not a result of cytotoxicity.

Inflammatory disorders are characterized, among other events, by the production of significant quantities of free radicals, nitrogen reactive species and cytokines $(7,21,23-25)$. TNF- $\alpha$ and IL-1 $\beta$ are considered to be critical cytokines in the inflammatory cytokine network, and are produced as a result of endotoxin exposure (2-5). Furthermore, TNF- $\alpha$ and IL-1 $\beta$ are important for promoting the expression of iNOS, in addition to the production of other cytokines, such as IL-6 and IL-8 $(21,25)$. Therefore, suppressing the overproduction and activity of pro-inflammatory cytokines is necessary to reduce inflammation and its symptoms, and this method has proved to be successful in the treatment of certain inflammatory diseases, including rheumatoid arthritis, obesity, diabetes mellitus, cancer and atherosclerosis $(2,5,21,23)$. In the present study, it was demonstrated that TSG is able to significantly suppress TNF- $\alpha$ and IL- $1 \beta$ production. mRNA and protein expression levels of these cytokines were also significantly inhibited, suggesting that TSG exerts its anti-inflammatory effects by suppressing pro-inflammatory cytokines at the transcriptional level.

As NF- $\kappa B$ and MAPK signaling molecules are key regulators of a variety of genes involved in inflammatory responses $(8,25)$, the effects of TSG on the NF- $\kappa B$ and MAPK signaling pathways were investigated in LPS-induced RAW 264.7 macrophages, in order to further elucidate the underlying mechanisms of anti-inflammation. Under normal physiological conditions, NF- $\mathrm{kB}$ is localizes in the cytosol as an inactive heterodimer consisting of three subunits: p50, p65 and Iк-B. Upon its activation by various stimuli, including LPS, the Iк-B protein is phosphorylated and degraded, resulting in the liberation of NF- $\mathrm{kB}$ and enabling it to translocate to the nucleus $(8,25)$. The translocation of NF- $\kappa \mathrm{B}$ into the nucleus triggers the expression of target genes, including pro-inflammatory cytokines, adhesion molecules, chemokines and inducible enzymes, such as iNOS $(26,27)$. The production of the aforementioned genes acts as a positive autocrine feedback signal to augment NF- $\mathrm{KB}$ activation, and subsequently increases the production of these pro-inflammatory factors $(7,8,28)$. The results of the present study demonstrated that the translocation of the p65 subunit of NF- $\mathrm{kB}$ to the nucleus, in addition to the degradation of I $\kappa \mathrm{B}-\alpha$, was markedly inhibited by pretreatment with TSG. Thus, the data indicated that TSG may exert its anti-inflammatory activity through NF- $\mathrm{KB}$ activation; by suppressing the degradation of IкB- $\alpha$ and the subsequent translocation of NF- $\mathrm{kB}$ p65 from the cytosol to the nucleus in LPS-stimulated RAW 264.7 cells. The present data provided a basis for modeling the effects of TSG in animal models, in order to facilitate the development of nutraceutical products and anti-inflammatory drugs based on TSG.

The MAPK family is a group of serine and threonine kinases that are activated in response to diverse extracellular stimuli to mediate signal transduction from the cell surface to the nucleus (29). Upon the activation of MAPKs, transcription factors that are present in the cytoplasm or nucleus are phosphorylated and activated. This activation leads to the expression of target genes that regulate cell growth, division, and/or differentiation $(30,31)$. As described previously, in the LPS-stimulated signaling pathway of macrophages, MAPKs have demonstrated an association with LPS-stimulated iNOS and pro-inflammatory cytokine expressions $(9,10)$. In the present study, it was identified that the phosphorylation levels of three MAPKs, ERK, JNK and p38 MAPK, were markedly increased in LPS-stimulated RAW 264.7 cells, compared with unstimulated cells; however, following TSG administration, the phosphorylation levels of the MAPKs markedly reduced in a dose-dependent manner. Therefore, it is postulated that attenuation of the phosphorylation levels of MAPKs by TSG may contribute to the inhibition of inflammatory reactions in LPS-stimulated RAW 264.7 cells.

In conclusion, TSG has the ability to inhibit the production of inflammatory cytokines, in addition to NO, in LPS-stimulated RAW 264.7 macrophages, by modulating the activation of intracellular signaling pathways, including NF- $\kappa \mathrm{B}$ and multiple MAPK signaling pathways. Thus, TSG may be a potential candidate for the treatment of various 
inflammatory diseases that are associated with the over-activation of macrophages.

\section{Acknowledgements}

The present study was supported by Blue-Bio Industry Regional Innovation Center at Dongeui University (Busan, Korea), as an RIC program under the Ministry of Trade, Industry and Energy and Busan City (RIC; grant no. RIC08-06-07), and the Basic Science Research Program through a National Research Foundation of Korea grant funded by the Korea government (grant no. 2015R1A2A2A01004633).

\section{References}

1. Zhang X and Mosser DM: Macrophage activation by endogenous danger signals. J Pathol 214: 161-178, 2008.

2. Fujihara M, Muroi M, Tanamoto K, Suzuki T, Azuma H and Ikeda H: Molecular mechanisms of macrophage activation and deactivation by lipopolysaccharide: Roles of the receptor complex. Pharmacol Ther 100: 171-194, 2003.

3. Park BS and Lee JO: Recognition of lipopolysaccharide pattern by TLR4 complexes. Exp Mol Med 45: e66, 2013.

4. Kapadia M and Sakic B: Autoimmune and inflammatory mechanisms of CNS damage. Prog Neurobiol 95: 301-333, 2011.

5. Laveti D, Kumar M, Hemalatha R, Sistla R, Naidu VG, Talla V, Verma V, Kaur N and Nagpal R: Anti-inflammatory treatments for chronic diseases: A review. Inflamm Allergy Drug Targets 12: 349-361, 2013

6. Muralidharan S and Mandrekar P: Cellular stress response and innate immune signaling: Integrating pathways in host defense and inflammation. J Leukoc Biol 94: 1167-1184, 2013.

7. Guha M and Mackman N: LPS induction of gene expression in human monocytes. Cell Signal 13: 85-94, 2001.

8. Li Q and Verma IM: NF-kappaB regulation in the immune system. Nat Rev Immunol 2: 725-734, 2002.

9. Caivano M: Role of MAP kinase cascades in inducing arginine transporters and nitric oxide synthetase in RAW264 macrophages. FEBS Lett 429: 249-253, 1998.

10. Uto T, Fujii M and Hou DX: 6-(Methylsulfinyl)hexyl isothiocyanate suppresses inducible nitric oxide synthase expression through the inhibition of Janus kinase 2-mediated JNK pathway in lipopolysaccharide-activated murine macrophages. Biochem Pharmacol 70: 1211-1221, 2005.

11. Choi J, Kim TH, Choi TY and Lee MS: Ginseng for health care: A systematic review of randomized controlled trials in Korean literature. PLoS One 8: e59978, 2013.

12. Coleman CI,Hebert JH and Reddy P: The effects of Panax ginseng on quality of life. J Clin Pharm Ther 28: 5-15, 2003.

13. Kitts D and Hu C: Efficacy and safety of ginseng. Public Health Nutr 3 (4A): 473-485, 2000.
14. Shibata S: Chemistry and cancer preventing activities of ginseng saponins and some related triterpenoid compounds. J Korean Med Sci 16: S28-37, 2001.

15. Chen RJ, Chung TY, Li FY, Lin NH and Tzen JT: Effect of sugar positions in ginsenosides and their inhibitory potency on $\mathrm{Na}^{+} / \mathrm{K}^{+}$-ATPase activity. Acta Pharmacol Sin 30: 61-69, 2009.

16. Lü JM, Yao Q and Chen C: Ginseng compounds: An update on their molecular mechanisms and medical applications. Curr Vasc Pharmacol 7: 293-302, 2009.

17. Kim JY, Lee HJ, Kim JS and Ryu JH: Induction of nitric oxide synthase by saponins of heat-processed ginseng. Biosci Biotechnol Biochem 69: 891-895, 2005.

18. Park JS, Park EM, Kim DH, Jung K, Jung JS, Lee EJ, Hyun JW, Kang JL and Kim HS: Anti-inflammatory mechanism of ginseng saponins in activated microglia. J Neuroimmunol 209: 40-49, 2009.

19. Kang A, Hao H, Zheng X, Liang Y, Xie Y, Xie T, Dai C, Zhao Q, Wu X, Xie L and Wang G: Peripheral anti-inflammatory effects explain the ginsenosides paradox between poor brain distribution and anti-depression efficacy. J Neuroinflammation 8: 100, 2011.

20. Sugimoto S, Nakamura S, Matsuda H, Kitagawa $\mathrm{N}$ and Yoshikawa M: Chemical constituents from seeds of Panax ginseng: Structure of new dammarane-type triterpene ketone, panaxadione, and hplc comparisons of seeds and flesh. Chem Pharm Bull (Tokyo) 57: 283-287, 2009.

21. Dinarello CA: Proinflammatory cytokines. Chest 118: 503-508, 2000.

22. Koppula S, Kumar H, Kim IS and Choi DK: Reactive oxygen species and inhibitors of inflammatory enzymes, NADPH oxidase, and iNOS in experimental models of Parkinson's disease. Mediators Inflamm 823902: 2012, 2012

23. Martinon F: Signaling by ROS drives inflammasome activation. Eur J Immunol 40: 616-619, 2010.

24. Korbecki J, Baranowska-Bosiacka I, Gutowska I and Chlubek D: The effect of reactive oxygen species on the synthesis of prostanoids from arachidonic acid. J Physiol Pharmacol 64: 409-421, 2013.

25. Marcus JS, Karackattu SL, Fleegal MA and Sumners C: Cytokine-stimulated inducible nitric oxide synthase expression in astroglia: role of Erk mitogen-activated protein kinase and NF-kappaB. Glia 41: 152-160, 2003.

26. Mercurio $F$ and Manning AM: NF-kappaB as a primary regulator of the stress response. Oncogene 18: 6163-6171, 1999.

27. Klemm S and Ruland J: Inflammatory signal transduction from the Fc epsilon RI to NF-kappa B. Immunobiology 211: 815-820, 2006.

28. Sonis ST: The biologic role for nuclear factor-kappaB in disease and its potential involvement in mucosal injury associated with anti-neoplastic therapy. Crit Rev Oral Biol Med 13: 380-389, 2002.

29. Kim EK and Choi EJ: Pathological roles of MAPK signaling pathways in human diseases. Biochim Biophys Acta 1802: 396-405, 2010.

30. Huang P, Han J and Hui L: MAPK signaling in inflammation-associated cancer development. Protein Cell 1: 218-226, 2010.

31. Kaminska B, Gozdz A,Zawadzka M, Ellert-Miklaszewska A and LipkoM: MAPK signal transduction underlying brain inflammation and gliosis as therapeutic target. Anat Rec (Hoboken) 292: 1902-1913, 2009. 